Please cite this paper as follows:

Hong-Ki Hong, Chein-Shan Liu, Ya-Po Shiao, and Bing-Chang Shih, Planar Double-Slip Model for Polycrystal Plasticity and Micro Tension Tests of Pure Nickel and Copper, ASME Journal of Engineering Materials and Technology, Vol.124, pp.314-321, 2002. 


\author{
Hong-Ki Hong \\ Department of Civil Engineering, \\ Taiwan University, \\ Taipei, Taiwan \\ Chein-Shan Liu \\ Department of Mechanical and Marine \\ Engineering, \\ Taiwan Ocean University, \\ Keelung, Taiwan \\ Ya-Po Shiao \\ Science and Technology Information Center, \\ National Science Council, \\ Taipei, Taiwan \\ Bing-Chang Shih \\ Department of Civil Engineering, \\ Taiwan University, \\ Taipei, Taiwan
}

\section{Planar Double-Slip Model for Polycrystal Plasticity and Micro Tension Tests of Pure Nickel and Copper}

\begin{abstract}
Many natural minerals and manmade materials are aggregates of crystals or polycrystalline solids with a nonrandom distribution of grain orientations. Macroscopic behavior in such textured polycrystals depends on directions and is thus anisotropic. In this paper we develop experimental and theoretical procedures for investigating grain orientation evolution and its effect on the tensile stress-strain curve. The micro-tensile experiments were executed in a self-developed micro-forcing-heating device together with a microrecorder-image analyzer system. In the experiments the $0.1 \mathrm{~mm}$ thin foil specimens of pure nickel and copper were gradually loaded toward final failure and the evolution of grain boundaries and slip bands inside grains was observed and recorded digitally via microscope and CCD camera throughout the whole time history. The texture image data were then used in a theoretical micro-macro transformation procedure to simulate the orientation evolutions and the stress-strain curves. The procedure was based on a double-slip model of polycrystal plasticity and on averaging of polycrystalline behavior over all grain orientations weighted by an orientation distribution function. The comparisons made between the simulated and experimental data of orientation evolutions and between the simulated curves and the macro-curves concurrently obtained in the experiments confirm the proposed procedures capable of simulating the considered micro-macro relations.

[DOI: $10.1115 / 1.1479355]$
\end{abstract}

[DOI: 10.1115/1.1479355]

\section{Introduction}

The processes to determine the macro responses of polycrystalline materials from their micro-constitutents are very complex. The main reason is that polycrystals usually exhibit anisotropy at large deformations due to preferred orientations of crystal grains. For any postulated micro-mechanism a fair amount of averaging must be taken before the macroscopic properties of the material element can be understood and predicted. Indeed, several known books in this realm, for example, Havner [1], Kocks et al. [2], and Meyers et al. [3], have provided an extensive overview of the anisotropic properties from the perspective of crystal plasticity, and a number of theoretical polycrystalline plasticity models, for example, Asaro [4], Harren [5], and Dluzewski [6], have been developed to deal with plastic behavior of polycrystals at large deformation and the accompanying texture evolution. In the recent decade a variety of double-slip models were proposed and combined with the averaging technique of orientation distribution function [7-11].

It is of significant interest to be able to visualize and digitally record the dynamical process of microstructural evolution and it is doubtless difficult to make concurrent measurement of external load and local strain histories in grains of polycrystalline metals. References [12,13] made related but somewhat different efforts. Saotome and Iguchi [12] developed an apparatus for observing changes of microstructure under varying temperatures but the mechanical loading was held constant statically. Traub et al. [13] developed a device for observing the process of generation of slip systems in a single crystal.

Possible mechanical testing techniques are (i) tension test, (ii) bulge test, and (iii) hardness test. Advantages and liabilities of the

Contributed by the Materials Division for publication in the JOURNAL OF ENGINEERING MATERIALS AND TECHNOLOGY. Manuscript received by the Materials Division September 3, 2001; revised manuscript received February 18, 2002. Guest Editors: Tariq A. Khraishi and Hussein M. Zbib. three techniques are summarized in [14]. Possible methods of manufacturing foil specimens are (i) (mechanical) cutting with a sharp blade [14-16], (ii) spark (thermal) cutting by a shaped electrode $[14,16]$, (iii) chemical etching with a mask [14,16], and (iv) electrochemical etching with a mask. ASTM [15] recommends that "The machined specimens shall be examined under about 20X magnification to determine that the edges are smooth and that there are no surface scratches or creases. Specimens showing discernible scratches, creases or edge discontinuities shall be rejected."

In this paper we develop both the experimental and theoretical procedures to investigate microstructural evolution and its effect on the macroscopic behavior. The experiments were executed in a micro-forcing-heating device together with a micro-recorderimage analyzer system. In Section 2 we focus on an experimental study through the self-developed device, which has been improved from an original design by Hong and Cheng [17]. In the tests the foil specimens of pure nickel and copper were gradually loaded toward final failure and the evolution of the surfaces of the specimens including grain boundaries and slip bands inside grains were simultaneously observed and recorded through microscope and CCD camera. In Section 3 we briefly summarize the formulas of a double-slip model for transforming the micro-quantities to the macro ones by averaging with respect to the orientation distribution function of all grains. The comparisons of the experimental data with the theoretical results are then conducted in Section 4, and finally, concluding remarks are made in Section 5. Although both nickel and copper tested herein are three-dimensional (3D) face-centered cubic (FCC) crystals with 12 slip systems, we limit our present scope in the theoretical aspect to a two-dimensional plane-stress model with only 2 active slip systems and in the image part of the experimental aspect, to surface images of the 0.1 $\mathrm{mm}$ thin foil specimens, ${ }^{1}$ and try to see at least whether a corre- 


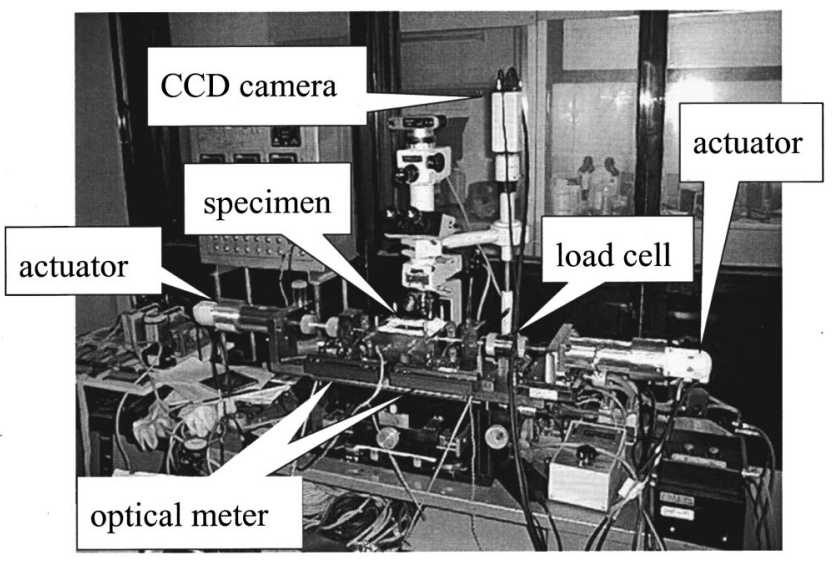

Fig. 1 The micro-forcing-heating device together with the micro-recorder-image analyzer system

lation can be established in this limited scope before a more sophisticated study is attempted.

\section{Experiments}

Figure 1 displays a self-developed micro-forcing-heating device as well as a microscope-recorder-image analyzer system, with which the whole loading and strain histories along with the images of microstructural evolution in a polycrystal can be recorded concurrently. One thereby visualizes the whole dynamical process from the original state with or without annealing, through the formation of slip systems, localization of plastic flow, cracking, to final failure. Specifically reported herein are two tests on specimens of high purity $(4 \mathrm{~N})$ nickel $(\mathrm{Ni})$ and copper $(\mathrm{Cu})$.

Since in our approach the tension test was selected for testing metal foils, to avoid edge effects, the shape of the specimen was designed to be edged smoothly and completely free from all forms of notches or other "stress raiser." To further avoid local zigzag in the edge, our specimens were prepared by a double-sided electrochemical etching with a precarved mask. A micrograph with about 80X magnification showed that the edges were smooth and that there were no discernible surface scratches. Indeed, the other three preparation methods fail to produce a usable foil specimen with such small size, shown in Fig. 2, as needed in our study.

The chemical composition analyses of the virgin metal foils $(50 \mathrm{~mm} \times 50 \mathrm{~mm} \times 0.125 \mathrm{~mm}, 4 \mathrm{~N})$ as received are shown in Table 1. The procedure of specimen preparation is given as follows. In the first step, the two surfaces of the foil were ground with 0.05 $\mu \mathrm{m} \mathrm{Al}_{2} \mathrm{O}_{3}$ powder until the surfaces looked like mirrors.

In the second step, the metal foil was coated with solid photoresistant, which was rolled onto the two surfaces of the metal foil, yielding a thin and continuous coating. The hot roller at about $80^{\circ} \mathrm{C}$ made the thin coating glued to the mirror-flat surfaces. The coated foil was mounted between the two image plate-mask and suitable exposure was made. The foil was then immersed in $\mathrm{Na}_{2} \mathrm{CO}_{3}$ solution for about 20 seconds or longer for developing and then in electrolytic solution (mixture solution of $\mathrm{H}_{3} \mathrm{BO}_{3}$, $\mathrm{NiCl}_{2}$ and $\mathrm{NiSO}_{4}$ for nickel specimens; $\mathrm{CuSO}_{4}$ solution for copper specimens) of $60-70^{\circ} \mathrm{C}$ for about 60 seconds for electrochemical etching. The double-faced etched foil was cleaned up with de-ionic (DI) water and then immersed in $\mathrm{NaOH}$ solution for

${ }^{1}$ Since a genuine $3 \mathrm{D}$ experimental study is difficult if not impossible, one may instead choose to explore the surfaces of the specimens on the basis of the assumption that there exists a certain projection operator, which may be a scalar factor or a matrix in simpler situations or a differential or integral operator in more complex situations and represents the physical relations between the body and the surface of the polycrystalline specimen under imposed experimental conditions.

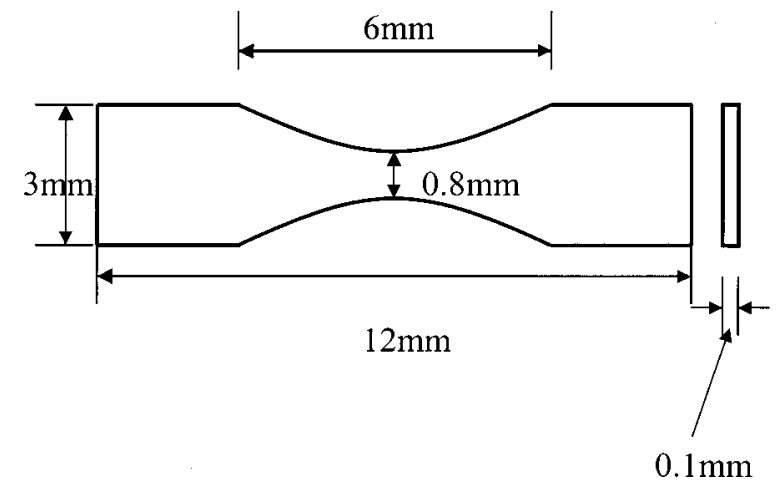

Fig. 2 Geometry of the foil specimen

about 15 seconds for stripping the solid coating from its surfaces. The stripped foil was cleaned up again with DI water.

To investigate the interaction between grains and grainboundaries, the observed surface of the specimen has to be etched. A solution of $\mathrm{FeCl}_{3}, 36 \mathrm{~g} / \mathrm{l}, 50^{\circ} \mathrm{C}, 3$ to 5 seconds is recommended for etching both nickel and copper foils.

A picture of the experimental apparatus is shown in Fig. 1, which contains four main sets. The first set is a pair of electromagnetic actuators, which have similar performance as to achieve symmetrical straining of the specimen. Otherwise, the image grabbed would not be on the same spot and the subsequent microstructural image analyses would not be conducted on the same area around the middle section of the specimen under the microscope. The second set is the digital CCD camera which is the most important part of the microscope-recorder-image analyzer system. The system is very powerful in grabbing and analyzing the microstructural image. The third set is a load cell that is used to measure the applying load. The fourth is a pair of optical meters that is used to measure the elongation of the specimen. With this system, the microstructural evolution during straining can be observed directly on an optical microscope and simultaneously transferred through the digital CCD camera to be displayed on a monitor and recorded in the computer hard disks. Thus the microscoperecorder-image analyzer system can grab and analyze the recorded image. In its present status, the time resolution of an image frame is $0.1-1$ second. The actuators can move the tension bar at speed $0.1 \mathrm{~mm} / \mathrm{min}$ in stroke control.

At high magnification deformation inside grains under the tension test can be measured in details, whereas the interaction between grains and grain-boundaries may be grabbed under medium magnification. However, if the interest is in the whole neck of the specimen, lower magnification is recommended, under which an overall picture of the evolution of microstructural deformation, deterioration, and fracture can be obtained.

\section{Theoretical Foundation}

The planar double-slip model, as has been used to model the behavior of polycrystals in the literature [7-11], assumes two slip systems coexistent in the deformation process for each grain. In this section we would not go to the details of the derivations which are relegated to the paper [18], but briefly summarize the

Table 1 Chemical compositions of nickel and copper (ppm)

\begin{tabular}{|c|c|c|c|c|c|c|c|c|c|}
\hline \hline Pure nickel & $\mathrm{Ag}$ & $\mathrm{Al}$ & $\mathrm{Ca}$ & $\mathrm{Cr}$ & $\mathrm{Cu}$ & $\mathrm{Fe}$ & $\mathrm{Mg}$ & $\mathrm{Mn}$ & $\mathrm{Si}$ \\
\hline & $<1$ & $<1$ & $<1$ & $<1$ & $<1$ & 20 & $<1$ & $<1$ & $<1$ \\
\hline Pure copper & $\mathrm{Ag}$ & $\mathrm{Al}$ & $\mathrm{Bi}$ & $\mathrm{Ca}$ & $\mathrm{Mg}$ & $\mathrm{Na}$ & $\mathrm{Pb}$ & $\mathrm{Si}$ & $\mathrm{Sn}$ \\
\hline & 50 & $<1$ & 1 & $<1$ & $<1$ & $<1$ & 3 & 2 & 1 \\
\hline \hline
\end{tabular}




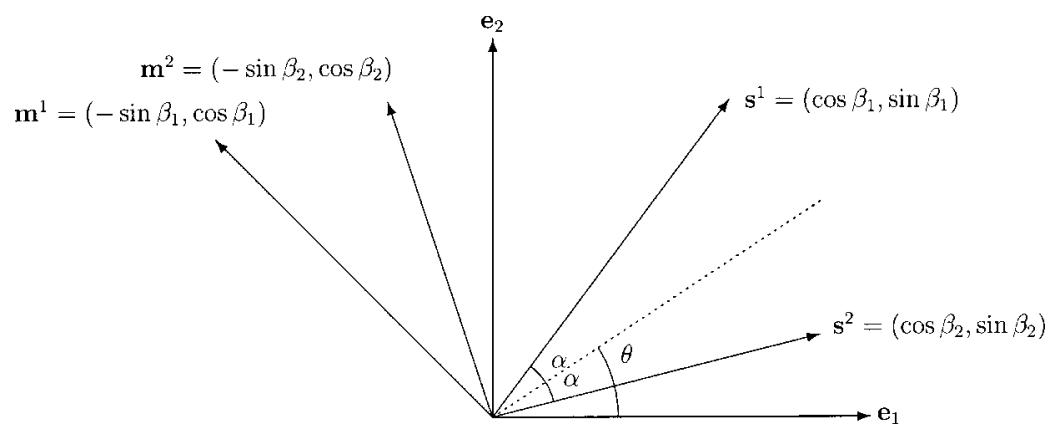

Fig. 3 Configuration of the planar double-slip model for a single crystal. $e_{1}$ is in the longitudinal (tensile) direction of the specimen.

required main results for the calculations of planar double-slip model, whose geometric description of the slip configuration is shown in Fig. 3. The two unit vectors associated with the slip direction and the normal direction of the slip plane are, respectively,

$$
\mathbf{s}^{(i)}=\left(\cos \beta_{i}, \sin \beta_{i}\right), \quad \mathbf{m}^{(i)}=\left(-\sin \beta_{i}, \cos \beta_{i}\right), \quad i=1,2,
$$

in which $\beta_{1}:=\theta+\alpha$ and $\beta_{2}:=\theta-\alpha$ with $\theta$ being the grain orientation and $2 \alpha$ the angle between the two slip directions $\mathbf{s}^{(1)}$ and $\mathbf{s}^{(2)}$.

The plastic deformation rate and plastic spin are calculated, respectively, by

$$
\mathbf{D}^{p}=\sum_{i=1}^{2} \dot{\gamma}^{(i)} \mathbf{P}^{(i)}, \quad \mathbf{W}^{p}=\sum_{i=1}^{2} \dot{\gamma}^{(i)} \Omega^{(i)},
$$

where $\dot{\gamma}^{(i)}, i=1,2$, are the slip rates of the two slip systems. In the above $\mathbf{P}^{(i)}$ and $\boldsymbol{\Omega}^{(i)}$ are the symmetric and skew-symmetric parts of the Schmid orientation tensor $\mathbf{s}^{(i)} \otimes \mathbf{m}^{(i)}$. The relation between the slip rates $\dot{\gamma}^{(i)}, i=1,2$, and the rates of Schmid resolved shear stresses $\dot{\tau}^{(i)}, i=1,2$, are realized through the hardening law of crystals:

$$
\left[\begin{array}{c}
\dot{\tau}^{(1)} \\
\dot{\tau}^{(2)}
\end{array}\right]=\mathbf{h}\left[\begin{array}{c}
\dot{\gamma}^{(1)} \\
\dot{\gamma}^{(2)}
\end{array}\right] .
$$

The hardening modulus tensor $\mathbf{h}$ has been discussed in several places, for example, Havner [1], Asaro [4], and Anand p. 236 of [3], and recognized to be the least well-characterized part of the constitutive models for crystal elastoplasticity.

To realize the micro-macro transformation, we average the grain stresses $\tau_{i j}$ over all orientations $\theta$ to obtain the overall stresses

$$
\sigma_{i j}=\frac{1}{\pi} \int_{-\pi / 2}^{\pi / 2} \tau_{i j}(\theta, t) f(\theta, t) d \theta, \quad i, j=1,2,
$$

in which $f(\theta, t)$, known as the orientation distribution function (ODF), plays the role of a weighting function. The governing equation of the ODF $f(\theta, t)$ is a first-order linear partial differential equation [19]:

$$
\frac{\partial f}{\partial t}+\dot{\theta} \frac{\partial f}{\partial \theta}+f \frac{\partial \dot{\theta}}{\partial \theta}=0
$$

which is subjected to the normalization condition:

$$
\frac{1}{\pi} \int_{-\pi / 2}^{\pi / 2} f(\theta, t) d \theta=1 .
$$

Figure 4 illustrates schematically the evolution of $f(\theta, t)$, where grains initially with random orientation distribution (i.e., $f(\theta, 0)$ $=1$ for all $\theta$ for the initial texture at $t=0$ ) in an annealed state evolves to have a nonuniform ODF (i.e., $f(\theta, t) \neq 1$ and varying with the orientation $\theta$ for the current texture at the current time $t$ ) under external loading.

The grain orientation $\theta$ is governed by the following equation [7]:

$$
\dot{\theta}=\frac{1}{2}\left(\dot{\gamma}^{(1)}+\dot{\gamma}^{(2)}\right)-W_{12}
$$

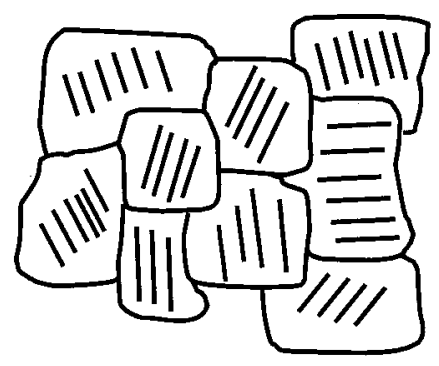

\section{Grains with different orientations}
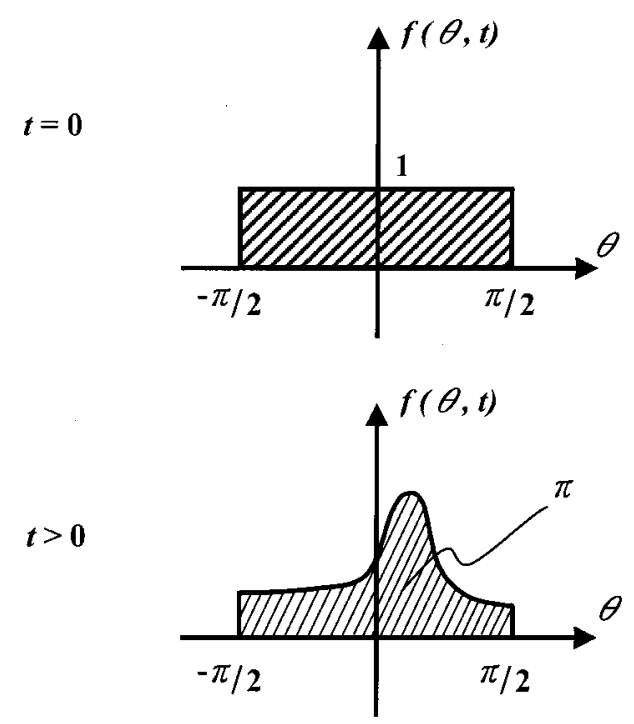

$f(\theta, t)$ is orientation distribution function (ODF)

Fig. 4 Under external loading a polycrystalline material which initially has a random distribution of grain orientations exhibits a certain texture with an evolving nonuniform ODF 
which, upon considering the slip rates, becomes [18]

$$
2 \dot{\theta}=b_{0} \cos 2 \theta+c_{0} \sin 2 \theta-2 W_{12},
$$

where $W_{12}=-W_{21}$ is the only nonzero component of the spin tensor $\mathbf{W}$, and

$$
\begin{aligned}
\xi:= & \frac{\eta:=\cos 4 \alpha}{\mu^{2} \sin ^{4} 4 \alpha+\mu\left(h_{12}+h_{21}\right)\left(\eta^{3}-\eta\right)+\left(1-\eta^{2}\right)[\mu \operatorname{trh}+\operatorname{det} \mathbf{h}]}, \\
b_{0}:= & \xi\left[\left(1-\eta^{2}\right) \mu+h_{11}-\eta h_{21}+\eta h_{22}-h_{12}\right]\left(\frac{D_{11}-D_{22}}{2} \cos 2 \alpha\right. \\
& \left.+D_{12} \sin 2 \alpha\right)-\xi\left[\left(1-\eta^{2}\right) \mu+h_{22}-\eta h_{12}+\eta h_{11}-h_{21}\right] \\
& \times\left(\frac{D_{11}-D_{22}}{2} \cos 2 \alpha-D_{12} \sin 2 \alpha\right), \\
c_{0}:= & -\xi\left[\left(1-\eta^{2}\right) \mu+h_{11}-\eta h_{21}+\eta h_{22}-h_{12}\right]\left(\frac{D_{11}-D_{22}}{2} \sin 2 \alpha\right. \\
& \left.-D_{12} \cos 2 \alpha\right)-\xi\left[\left(1-\eta^{2}\right) \mu+h_{22}-\eta h_{12}+\eta h_{11}-h_{21}\right] \\
& \times\left(\frac{D_{11}-D_{22}}{2} \sin 2 \alpha+D_{12} \cos 2 \alpha\right) .
\end{aligned}
$$

In the above $\mu$ is the shear modulus of the considered material, $D_{11}, D_{12}$, and $D_{22}$ are three independent components of the deformation rate tensor $\mathbf{D}$, and $h_{11}, h_{12}, h_{21}$, and $h_{22}$ are four independent components of the hardening modulus tensor $\mathbf{h}$. Up to now the equations needed to calculate the stress responses and grain orientations have been established.

Since our goal is to compare the theoretical results with the experimental data under certain loading conditions, which are naturally preferred to be as simple as possible, such that they can be experimentally realized easily and the corresponding theoretical solutions can be obtained in closed-form, we thus consider a constant hardening modulus tensor and let the velocity gradients be constant in time, ${ }^{2}$ consistent with the experimental condition executed herein which were under linear stroke control with velocity constant in time. With these constancy conditions $b_{0}$ and $c_{0}$ in Eqs. (11) and (12) become constant. Then Eq. (8) can be recast to

$$
2 \dot{\theta}=a_{0}\left[\cos \left(2 \theta-\beta_{0}\right)-\chi\right]
$$

with

$$
a_{0}:=\sqrt{b_{0}^{2}+c_{0}^{2}}, \quad \beta_{0}:=\arctan \frac{c_{0}}{b_{0}}, \quad \chi:=\frac{2 W_{12}}{a_{0}} .
$$

It deserves to point out that the parameter $\chi$ incorporates the independent effects of the slip system geometry, hardening law, and flow kinematics on the response of the microstructure and plays a central role in the criterion of the bifurcation behavior of Eq. (13).

Three possible cases of Eq. (13) must be considered separately, namely (1) $\chi>1$, (2) $\chi=1$, and (3) $\chi<1$. However, for saving space we consider only $\chi<1$, which is the case of our experiments and simulations, and write down directly the closed-form formulas for orientation, orientation distribution function, and overall stresses.

3.1 Closed-Form Solutions of Orientation and ODF. The orientation $\theta(t)$ and the $\operatorname{ODF} f(\theta, t)$ are given, respectively, by

$$
\begin{aligned}
& \qquad \theta(t)=\frac{\beta_{0}}{2}+\frac{1}{2} \arccos \left(\frac{1}{d_{1}+d_{2} \cos (}\right. \\
& f(\theta, t)=\frac{1}{a_{1}(t)+a_{2}(t) \cos \left(2 \theta-\beta_{0}\right)+a_{3}(t) \sin \left(2 \theta-\beta_{0}\right)} \\
& \text { In the above } \theta_{0} \text { denotes the initial value of } \theta, \text { and } \\
& d_{1}:=1+\frac{1-\chi \cos \left(2 \theta_{0}-\beta_{0}\right)}{\chi^{2}\left(1-\chi^{2}\right)}, \quad d_{2}:=\frac{\chi \cos \left(2 \theta_{0}-\beta_{0}\right)-1}{\chi^{2}\left(1-\chi^{2}\right)}, \\
& a_{1}(t):=1+\frac{1}{\chi^{2}\left(1-\chi^{2}\right)}\left[1-\cos \left(2 \sqrt{1-\chi^{2}} W_{12} t\right)\right] \\
& a_{2}(t):=\frac{\chi \sqrt{1-\chi^{2}}}{\chi\left(1-\theta_{0}^{2}\right)}\left[\cos \left(2 \sqrt{1-\chi^{2}} W_{12} t\right)-1\right] \\
& a_{3}(t):=\frac{-\sin \left(2 \sqrt{1-\chi^{2}} W_{12} t\right)}{\chi \sqrt{1-\chi^{2}}} .
\end{aligned}
$$

$$
\begin{aligned}
\sigma_{11}(t)= & \frac{1}{2}\left(\sigma_{11}(0)+\sigma_{22}(0)\right)+E^{*}\left(D_{11}+D_{22}\right) t \\
& +\frac{B_{1}(t) a_{2}(t)+C_{1}(t) a_{3}(t)}{a_{2}^{2}(t)+a_{3}^{2}(t)} \\
& -\frac{a_{1}(t)\left[B_{1}(t) a_{2}(t)+C_{1}(t) a_{3}(t)\right]}{a_{2}^{2}(t)+a_{3}^{2}(t)}-B_{2} I_{1}(t)-C_{2} I_{2}(t) \\
& +B_{2} I_{3}(t)+C_{2} I_{4}(t)+B_{3} I_{5}(t)+C_{3} I_{6}(t), \\
\sigma_{22}( & (t)=\sigma_{11}(0)+\sigma_{22}(0)+2 E^{*}\left(D_{11}+D_{22}\right) t-\sigma_{11}(t) \\
\sigma_{12}(t)= & \frac{B_{1}(t) a_{3}(t)-C_{1}(t) a_{2}(t)}{a_{2}^{2}(t)+a_{3}^{2}(t)} \\
& -\frac{a_{1}(t)\left[B_{1}(t) a_{3}(t)-C_{1}(t) a_{2}(t)\right]}{a_{2}^{2}(t)+a_{3}^{2}(t)}+C_{2} I_{1}(t)-B_{2} I_{2}(t) \\
& -C_{2} I_{3}(t)+B_{2} I_{4}(t)-C_{3} I_{5}(t)+B_{3} I_{6}(t),
\end{aligned}
$$

3.2 Closed-Form Solutions of Overall Stresses. Substituting Eq. (16) and the grain stresses into Eq. (4) and through a lot of calculations, we obtain the overall stresses as follows:

${ }^{2}$ Note that the velocity gradients were not at all assumed to be constant in space. It is reminded that in the current model the spatial dependence of all relevant quantities including the velocity gradients were taken into account by using the orientation distribution function. 


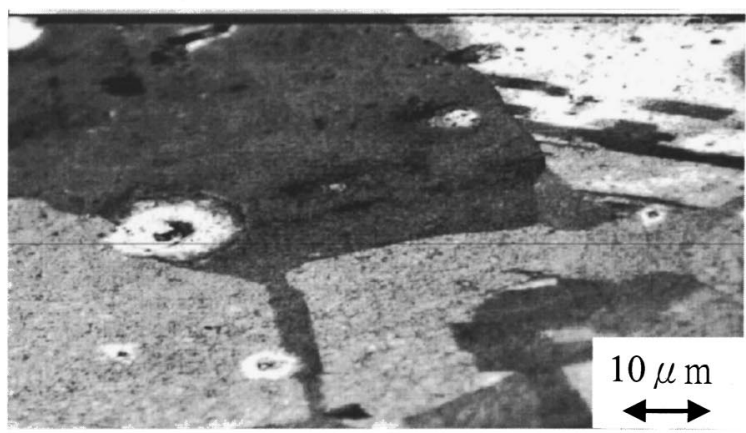

(a) 0 second $($ strain $=0)$

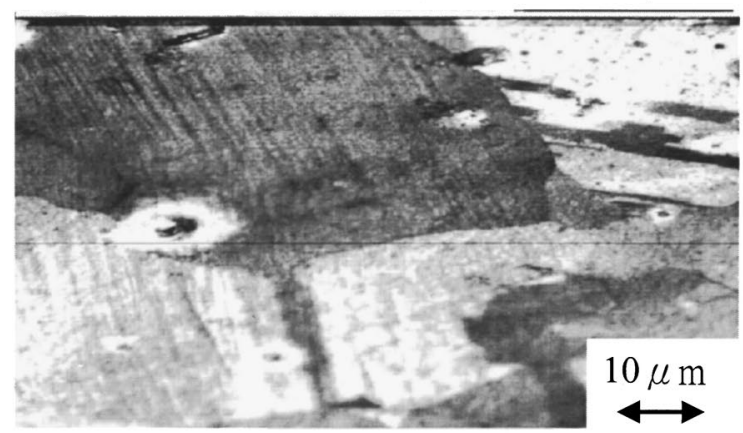

(b) 100 seconds (strain $=0.024)$

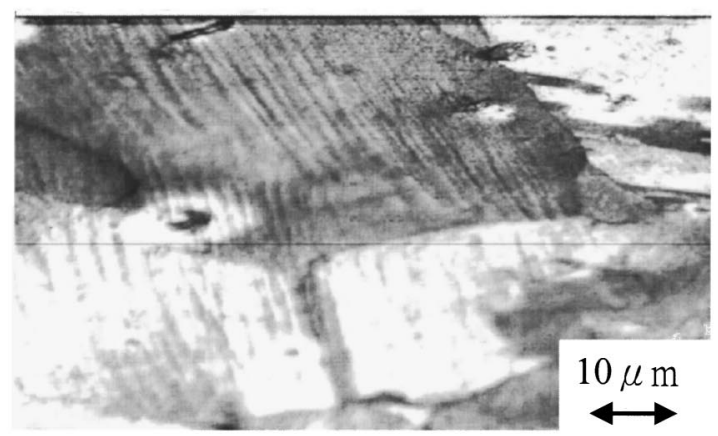

(c) 200 seconds (strain $=0.048$ )

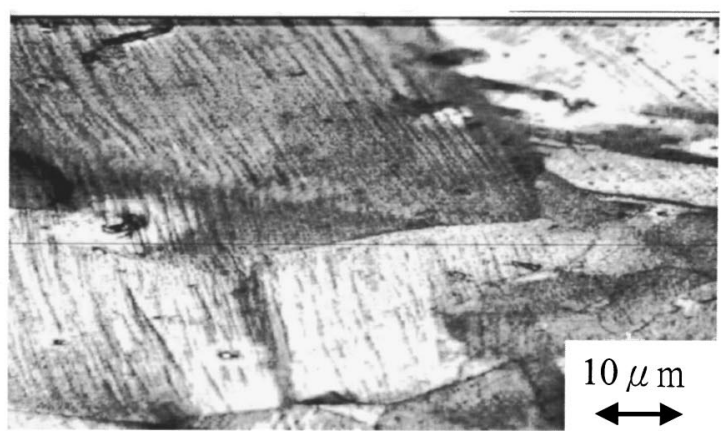

(d) 300 seconds (strain $=0.072$ )

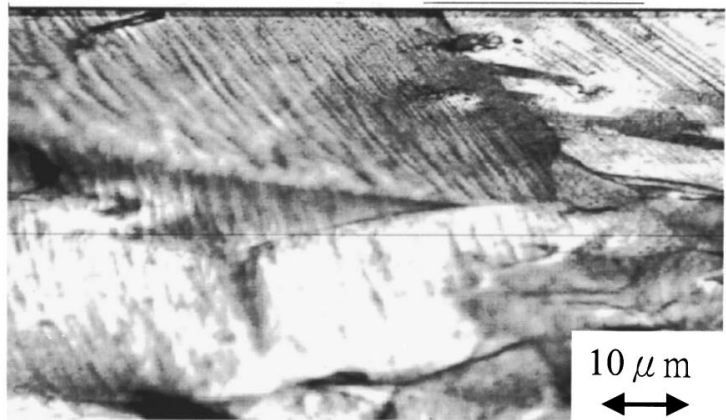

(e) 400 seconds ( strain $=0.096)$

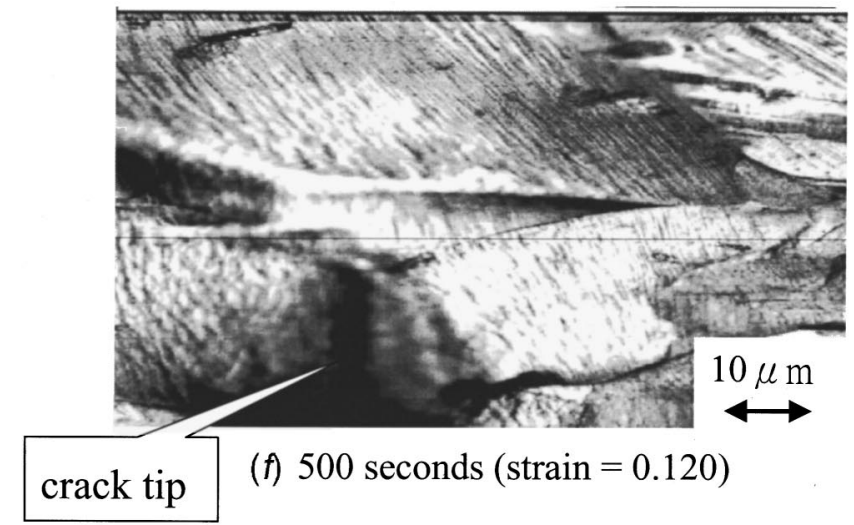

Fig. 5 Optical micrographs of deformation process of a nickel specimen in uniaxial tension: (a) original state at 0 second, $(b)$ at 100 seconds, (c) at 200 seconds, (d) at 300 seconds, (e) at 400 seconds, and ( $f$ ) at 500 seconds before the specimen ruptured

where

$$
E^{*}=\left\{\begin{array}{cc}
\frac{(3 \lambda+2 \mu) \mu}{\lambda+2 \mu} & \text { plane stress, } \\
\lambda+\mu & \text { plane strain. }
\end{array}\right.
$$

The $B_{i}, i=1,2,3, C_{i}, i=1,2,3$, and $I_{i}, i=1, \ldots, 6$, required by the above stress formulas are relegated to the Appendix. With these formulas we are able to simulate the macroscopic behavior based upon the micro-test data recorded in the micro-tension tests and compare the simulated results with the macroscopic test data.

\section{Experimental Results and Comparisons With Theo- retical Predictions}

Reported herein is an experimental work on the foil specimens of $4 \mathrm{~N}$ purity nickel and copper of the shape and dimensions as shown in Fig. 2. Although the whole time histories were recorded, to save space of presentation only six selected micrographs of a nickel specimen are shown in Fig. 5, demonstrating the dynamical deformation process. Figure 5(a) displays the original annealed state of the specimen. The grain sizes were 30-60 $\mu \mathrm{m}$ (for nickel, whereas $10-20 \mu \mathrm{m}$ for copper). ${ }^{3}$ Figures $5(b)-5(f)$ were taken consecutively at the equal time intervals (5@100 seconds). Figure $5(f)$ is the micrograph at 60 seconds prior to the rupture $(560$ seconds) of the specimen. It can be seen that strain localization appeared gradually (Figs. 5(b)-5(e)) and developed into a dominant crack (Fig. 5(f)). The dark area on the micrograph that can be observed was the tip of the crack. Notice that localization and subsequent cracking did occur in the middle of grains instead of between grains on grain-boundaries, and that the grains containing localization underwent apparent expansion. The parallel dark lines observed within grains were considered as slip bands, and thereby

${ }^{3}$ These amounts to 2-4 grains for nickel and 5-10 grains for copper in the thickness direction of the foil specimens. 


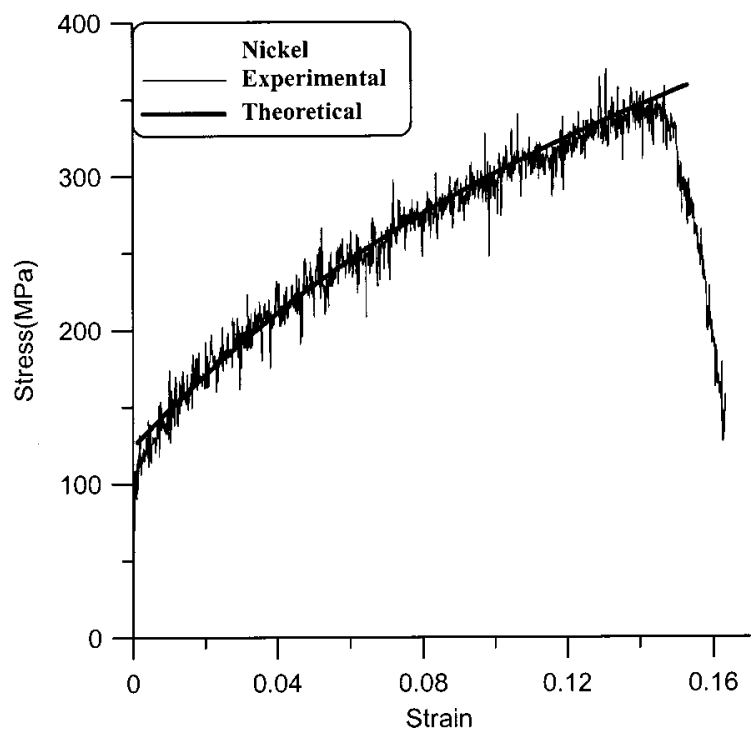

Fig. 6 Comparison of experimental macro data with theoretical simulation for the tensile stress-strain curve of nickel

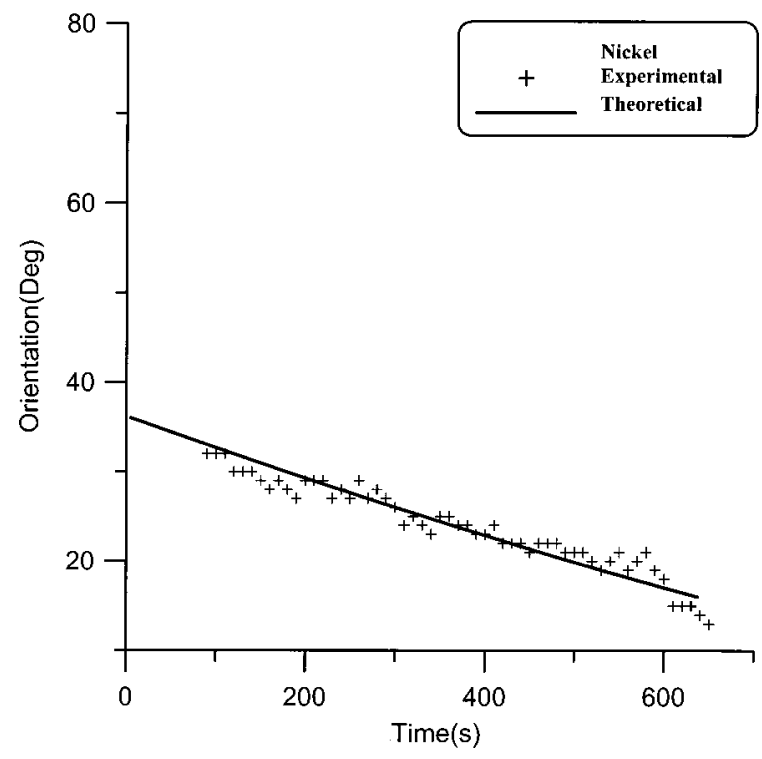

Fig. 7 Comparison of observed micro data with theoretical simulation for orientation evolution of nickel

slip orientations (and ODF) were measured and calculated via the micro-recorder-image analyzer system.

For nickel, the stress-strain relation of the simulated micromacro data and the macro-stress-strain curve obtained concurrently in the experiment are shown in Fig. 6, whereas the observed (and image analyzed) time history of orientation of a selected grain and the simulated time history of orientation of the single grain are shown in Fig. 7. In the above $D_{11}=2.39 \times 10^{-4} \mathrm{~s}^{-1}$ was specified for stroke control, $D_{22}=-D_{11}=-2.39 \times 10^{-4} \mathrm{~s}^{-1}$ was assumed and $W_{12}=-W_{21}=2.9 \times 10^{-4} \mathrm{~s}^{-1}$ were measured (and calculated) from the recorded microstructural image data, ${ }^{4}$ while all other $D_{i j}$ and $W_{i j}$ components are zeros, and the parameters $\mu=80000 \mathrm{MPa}, \nu=0.31, h_{11} / \mu=h_{22} / \mu=3 \times 10^{-2}$, and $h_{12} / \mu$ $=h_{21} / \mu=4 \times 10^{-2}$ were chosen in the model simulation.

${ }^{4}$ Three points on the images were identified and the angles subtended were traced continuously and their average time change rate was supposed to be $W_{12}$.
For copper, the stress-strain relation of the simulated micromacro data and the macro-stress-strain curve obtained concurrently in the experiment are shown in Fig. 8, whereas the observed (and image analyzed) time history of orientation of a selected grain and the simulated time history of orientation of the single grain are shown in Fig. 9. Here $D_{11}=-D_{22}=2.39 \times 10^{-4} \mathrm{~s}^{-1}$, $W_{12}=-W_{21}=3.8 \times 10^{-4} \mathrm{sec}^{-1}$, and all other $D_{i j}$ and $W_{i j} \mathrm{com}-$ ponents are zeros, while $\mu=42000 \mathrm{MPa}, \nu=0.35, h_{11} / \mu$ $=h_{22} / \mu=h_{12} / \mu=h_{21} / \mu=4 \times 10^{-2}$.

The values of the hardening moduli used above are slightly larger than that suggested by Asaro [4] for FCC materials. The parameter $\alpha$ was taken to be $\alpha=25 \mathrm{deg}$ for each material [9]. The values of the shear moduli $\mu$ and Poisson's ratios $\nu$ used above can be found in many metal handbooks. Those comparisons in Figs. 6-9 show that the proposed model can simulate the experimental data quite well. In these figures the solid black curves for stress were calculated from Eq. (19), and those for orientation were calculated from Eq. (15). The plus signs in Figs. 7 and 9

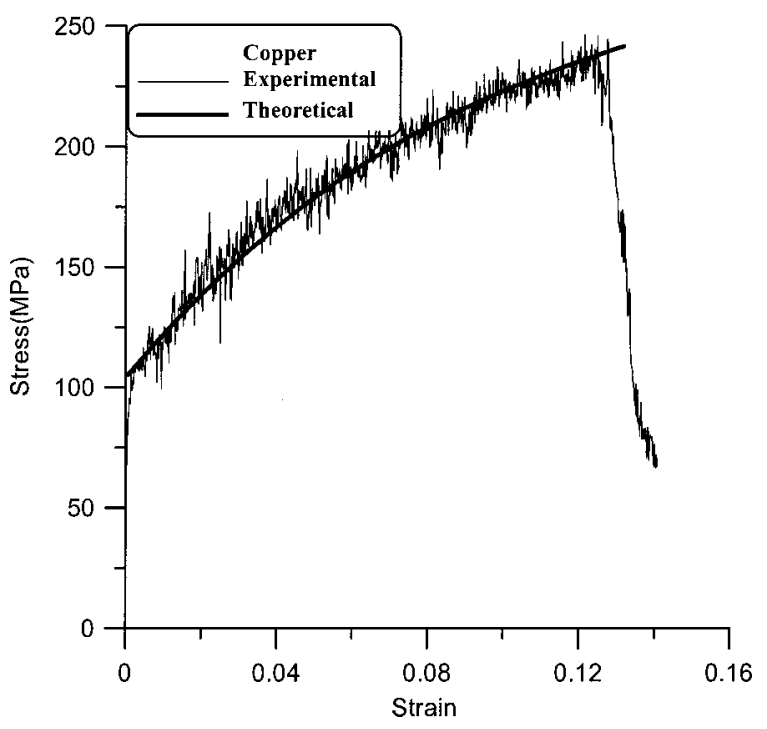

Fig. 8 Comparison of experimental macro data with theoretical simulation for the tensile stress-strain curve of copper

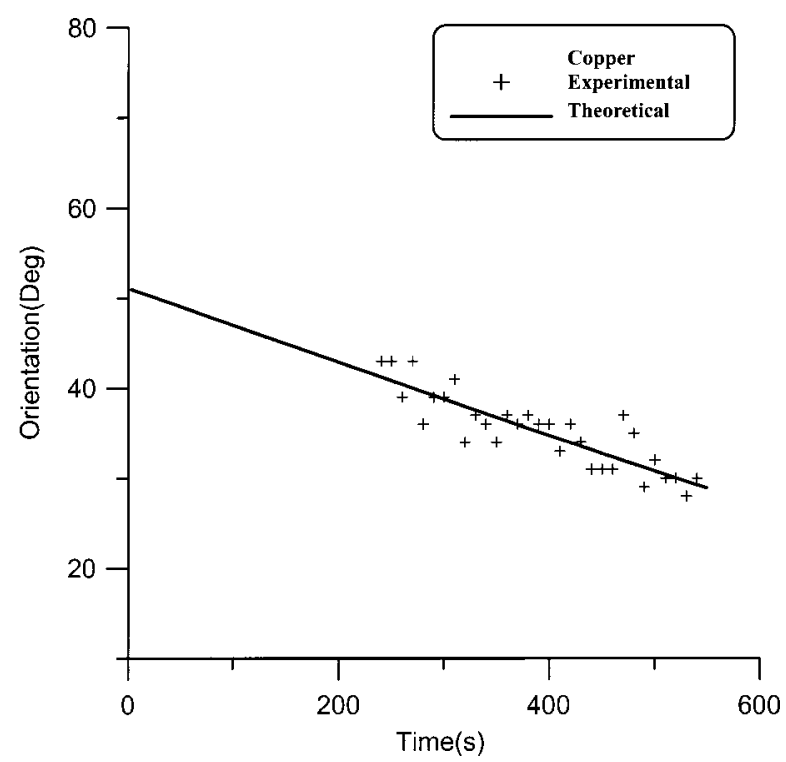

Fig. 9 Comparison of observed micro data with theoretical simulation for orientation evolution of copper 
represent the orientations measured and analyzed from the recorded microstructural images (by using the micro-recorder-image analyzer system), from which it was observed that the slip-line patterns that developed were not visible until slip bands emerged to surfaces at about 100 seconds for nickel and 200 seconds for copper.

\section{Concluding Remarks}

In this paper procedures were developed to concurrently record both the texture image data and macro experimental data and to perform the micro-macro transformation. The orientation averaging technique and the double-slip model were employed to simulate the tension test data of pure nickel and copper. The comparisons revealed that the simulated results from using experimental microstructural data in the model were in good agreement with the experimental macro stress-strain curves.

However, the planar assumption of the model made herein should not be overlooked. It seemed that the good agreement of the results justified the assumption, implying that the planar double-slip model together with the ODF of the scalar orientation sufficed to represent the mechanism of active slip planes and slip systems and that there existed a simpler projection operator between the surface image and the solid body of the foil specimen. The raised question may still be open until a 3D experimental technique and a 3D theoretical model of the micro-macro transformation are developed successfully and consistently. A less ambitious but more feasible direction for further work which can go well along with the experimental techniques presented here is to develop theoretically a full 3D model and its accompanying projection operator under the experimental conditions.

\section{Appendix}

In this Appendix we give $B_{i}, i=1,2,3, C_{i}, i=1,2,3$, and $I_{i}, i$ $=1, \ldots, 6$, and some initial conditions required in the stress formulas (19)-(21).

$$
\begin{aligned}
B_{1}(t):= & \frac{1}{\sin 4 \alpha}\left[\tau^{(2)}(0) \cos \left(\beta_{0}+2 \alpha\right)-\tau^{(1)}(0) \cos \left(\beta_{0}-2 \alpha\right)\right] \\
& +\frac{2\left(b_{1} b_{0}+c_{1} c_{0}\right) W_{12} t}{a_{0}^{2} \sin 4 \alpha}\left[h_{21} \cos \left(\beta_{0}+2 \alpha\right)\right. \\
& \left.-h_{11} \cos \left(\beta_{0}-2 \alpha\right)\right]+\frac{2\left(b_{2} b_{0}+c_{2} c_{0}\right) W_{12} t}{a_{0}^{2} \sin 4 \alpha} \\
& \times\left[h_{22} \cos \left(\beta_{0}+2 \alpha\right)-h_{12} \cos \left(\beta_{0}-2 \alpha\right)\right]
\end{aligned}
$$

$$
\begin{aligned}
C_{1}(t):= & \frac{1}{\sin 4 \alpha}\left[\tau^{(1)}(0) \sin \left(\beta_{0}-2 \alpha\right)-\tau^{(2)}(0) \sin \left(\beta_{0}+2 \alpha\right)\right] \\
& +\frac{2\left(b_{1} b_{0}+c_{1} c_{0}\right) W_{12} t}{a_{0}^{2} \sin 4 \alpha}\left[h_{11} \sin \left(\beta_{0}-2 \alpha\right)\right. \\
& \left.-h_{21} \sin \left(\beta_{0}+2 \alpha\right)\right]+\frac{2\left(b_{2} b_{0}+c_{2} c_{0}\right) W_{12} t}{a_{0}^{2} \sin 4 \alpha} \\
& \times\left[h_{12} \sin \left(\beta_{0}-2 \alpha\right)-h_{22} \sin \left(\beta_{0}+2 \alpha\right)\right]
\end{aligned}
$$

$$
\begin{aligned}
B_{2}:= & \frac{b_{1} b_{0}+c_{1} c_{0}}{a_{0}^{2} \sin 4 \alpha}\left[h_{21} \cos \left(\beta_{0}+2 \alpha\right)-h_{11} \cos \left(\beta_{0}-2 \alpha\right)\right] \\
& +\frac{b_{2} b_{0}+c_{2} c_{0}}{a_{0}^{2} \sin 4 \alpha}\left[h_{22} \cos \left(\beta_{0}+2 \alpha\right)-h_{12} \cos \left(\beta_{0}-2 \alpha\right)\right],
\end{aligned}
$$

$$
\begin{aligned}
C_{2}:= & \frac{b_{1} b_{0}+c_{1} c_{0}}{a_{0}^{2} \sin 4 \alpha}\left[h_{11} \sin \left(\beta_{0}-2 \alpha\right)-h_{21} \sin \left(\beta_{0}+2 \alpha\right)\right] \\
& +\frac{b_{2} b_{0}+c_{2} c_{0}}{a_{0}^{2} \sin 4 \alpha}\left[h_{12} \sin \left(\beta_{0}-2 \alpha\right)-h_{22} \sin \left(\beta_{0}+2 \alpha\right)\right],
\end{aligned}
$$

$$
\begin{aligned}
B_{3}:= & \frac{b_{1} c_{0}-c_{1} b_{0}}{a_{0}^{2} \sin 4 \alpha}\left[h_{21} \cos \left(\beta_{0}+2 \alpha\right)-h_{11} \cos \left(\beta_{0}-2 \alpha\right)\right] \\
& +\frac{b_{2} c_{0}-c_{2} b_{0}}{a_{0}^{2} \sin 4 \alpha}\left[h_{22} \cos \left(\beta_{0}+2 \alpha\right)-h_{12} \cos \left(\beta_{0}-2 \alpha\right)\right],
\end{aligned}
$$

$$
\begin{aligned}
C_{3}:= & \frac{b_{1} c_{0}-c_{1} b_{0}}{a_{0}^{2} \sin 4 \alpha}\left[h_{11} \sin \left(\beta_{0}-2 \alpha\right)-h_{21} \sin \left(\beta_{0}+2 \alpha\right)\right] \\
& +\frac{b_{2} c_{0}-c_{2} b_{0}}{a_{0}^{2} \sin 4 \alpha}\left[h_{12} \sin \left(\beta_{0}-2 \alpha\right)-h_{22} \sin \left(\beta_{0}+2 \alpha\right)\right],
\end{aligned}
$$




$$
I_{6}(t):=\frac{1}{2 \pi} \int_{-\pi-\beta_{0}}^{\pi-\beta_{0}} \frac{\sin \phi \ln \left(a_{1}(t)+a_{2}(t) \cos \phi+a_{3}(t) \sin \phi\right)}{a_{1}(t)+a_{2}(t) \cos \phi+a_{3}(t) \sin \phi} d \phi .
$$

The above resolved shear stresses $\tau^{(1)}(0)$ and $\tau^{(2)}(0)$ are given by

$$
\begin{gathered}
\tau^{(1)}(0)=\frac{\sigma_{22}(0)-\sigma_{11}(0)}{2} \sin \left(\theta_{0}+2 \alpha\right)+\sigma_{12}(0) \cos \left(\theta_{0}+2 \alpha\right), \\
\tau^{(2)}(0)=\frac{\sigma_{22}(0)-\sigma_{11}(0)}{2} \sin \left(\theta_{0}-2 \alpha\right)+\sigma_{12}(0) \cos \left(\theta_{0}-2 \alpha\right),
\end{gathered}
$$

and the other four constants are given by

$$
\begin{aligned}
b_{1}:= & \xi\left(\eta h_{22}-h_{12}\right)\left(\frac{D_{11}-D_{22}}{2} \cos 2 \alpha+D_{12} \sin 2 \alpha\right)-\xi\left(1+h_{22}\right. \\
& \left.-\eta h_{12}\right)\left(\frac{D_{11}-D_{22}}{2} \cos 2 \alpha-D_{12} \sin 2 \alpha\right), \\
c_{1}:= & \xi\left(\eta h_{22}-h_{12}\right)\left(\frac{D_{11}-D_{22}}{2} \sin 2 \alpha-D_{12} \cos 2 \alpha\right)-\xi\left(1+h_{22}\right. \\
- & \left.\eta h_{12}\right)\left(\frac{D_{11}-D_{22}}{2} \sin 2 \alpha+D_{12} \cos 2 \alpha\right), \\
b_{2}:= & \xi\left(1+h_{11}-\eta h_{21}\right)\left(\frac{D_{11}-D_{22}}{2} \cos 2 \alpha+D_{12} \sin 2 \alpha\right) \\
& -\xi\left(\eta h_{11}-h_{21}\right)\left(\frac{D_{11}-D_{22}}{2} \cos 2 \alpha-D_{12} \sin 2 \alpha\right), \\
c_{2}:= & -\xi\left(1+h_{11}-\eta h_{21}\right)\left(\frac{D_{11}-D_{22}}{2} \sin 2 \alpha-D_{12} \cos 2 \alpha\right) \\
& -\xi\left(\eta h_{11}-h_{21}\right)\left(\frac{D_{11}-D_{22}}{2} \sin 2 \alpha+D_{12} \cos 2 \alpha\right) .
\end{aligned}
$$

\section{Nomenclature}

$$
\begin{aligned}
\mathbf{s}^{(i)}, i=1,2= & i \text { th slip direction } \\
\mathbf{m}^{(i)}, i=1,2= & \text { normal direction of the } i \text { th slip plane } \\
\mathbf{s}^{(i)} \otimes \mathbf{m}^{(i)}= & \text { Schmid orientation tensor } \\
2 \alpha= & \text { angle between } \mathbf{s}^{(1)} \text { and } \mathbf{s}^{(2)} \\
\mathbf{e}_{i}, i=1,2= & \text { orthonormal base vectors, } \mathbf{e}_{1} \text { being in the ten- } \\
& \text { sile direction } \\
\theta= & \text { grain orientation } \\
\beta_{i}, i=1,2= & \text { angle between } \mathbf{s}^{(i)} \text { and } \mathbf{e}_{1} \\
f(\theta, t)= & \text { orientation distribution function }(\mathrm{ODF}) \\
\tau_{i j}, i, j=1,2= & \text { Kirchhoff stress components of grain } \\
\sigma_{i j}, i, j=1,2= & \text { overall stress components } \\
\mathbf{D}= & \text { deformation rate tensor } \\
\mathbf{W}= & \text { spin tensor } \\
\mathbf{D}^{p}= & \text { plastic deformation rate tensor } \\
\mathbf{W} & =\text { plastic spin tensor } \\
\mathbf{P}^{(i)}, i=1,2= & \text { symmetric part of the Schmid orientation ten- } \\
\mathbf{\Omega}^{(i)}, i=1,2= & \text { sor } \\
\dot{\gamma}^{(i)}, i=1,2= & \text { tensor }=\text { slip rate } \\
\dot{\tau}^{(i)}, i=1,2= & \text { rate of Schmid resolved shear stress } \\
\mathbf{h}= & \text { hardening modulus tensor }
\end{aligned}
$$

$\lambda, \mu, \nu=$ Lamé constants and the Poisson ratio

$E^{*}=$ material constant for plane stress or plane strain defined in Eq. (22)

$$
\eta=\cos 4 \alpha
$$

$\xi, b_{0}, c_{0}=$ constants defined, respectively, in Eqs. (10), (11), and (12)

$$
\begin{aligned}
a_{0} & =\sqrt{b_{0}^{2}+c_{0}^{2}} \\
\beta_{0} & =\arctan \left(c_{0} / b_{0}\right) \\
\chi & =2 W_{12} / a_{0}
\end{aligned}
$$

$d_{i}, i=1,2,3=$ constants defined in Eq. (17)

$a_{i}, i=1,2,3=$ time-dependent functions defined in Eq. (18)

$B_{1}, C_{1}=$ time-dependent functions defined, respectively, in Eqs. (A1) and (A2)

$B_{i}, i=2,3=$ constants defined respectively in Eqs. (A3) and (A5)

$C_{i}, i=2,3=$ constants defined respectively in Eqs. (A4) and (A6)

$I_{i}, i=1, \ldots, 6=$ time-dependent functions defined respectively in Eqs. (A7)-(A12)

$b_{i}, i=1,2=$ constants defined respectively in Eqs. $(A 15)$ and $(A 17)$

$c_{i}, i=1,2=$ constants defined respectively in Eqs. (A16) and (A18)

\section{References}

[1] Havner, K. S., 1992, Finite Plastic Deformation of Crystalline Solids, Cambridge University Press, Cambridge.

[2] Kocks, U. F., Tomé, C. N., and Wenk, H.-R., 1998, Texture and Anisotropy: Preferred Orientations in Polycrystals and their Effect on Materials Properties, Cambridge University Press, Cambridge.

[3] Meyers, M. A., Armstrong, R. W., and Kirchner, H. O. K., eds., 1999, Mechanics and Materials: Fundamentals and Linkages, Wiley, New York.

[4] Asaro, R. J., 1983, "Micromechanics of crystals and polycrystals," Advances in Applied Mechanics, Vol. 23, Hutchinson, J. W., and Wu, T. Y., eds., Academic Press, New York, pp. 1-115.

[5] Harren, S. V., 1997, "Theory of evolution of crystal lattice orientation density and state variables in Euler space," Int. J. Plast., 13, pp. 59-74.

[6] Dluzewski, P. H., 1991, "Crystal orientation spaces and remarks on the modelling of polycrystal anisotropy," J. Mech. Phys. Solids, 39, pp. 651-661.

[7] Rashid, M. M., 1992, "Texture evolution and plastic response of twodimensional polycrystals," J. Mech. Phys. Solids, 40, pp. 1009-1029.

[8] van der Giessen, E., and van Houtte, P., 1992, "A 2D analytical multiple slip model for continuum texture development and plastic spin," Mech. Mater., 13, pp. $93-115$.

[9] Dafalias, Y. F., 1993, "Planar double-slip micromechanical model for polycrystal plasticity,” J. Eng. Mech., 119, pp. 1260-1284.

[10] Prantil, V. C., Jenkins, J. T., and Dawson, P. R., 1993, “An analysis of texture and plastic spin for planar polycrystals," J. Mech. Phys. Solids, 41, pp. 13571382.

[11] Qian, Z., and Wu, H. C., 1996, “A 2-D texture study based on a double-slip model of polycrystal plasticity with analysis of thin-walled tubes under torsion," Int. J. Solids Struct., 33, pp. 4167-4193.

[12] Saotome, Y., and Iguchi, N., 1987, "In-situ microstructural observations and micro-grid analyses of transformation superplasticity in pure Fe,' ISIJ Int., 27, pp. 696-704.

[13] Traub, H., Neuhäuser, H., and Schwink, Ch., 1977, "Investigations of the yield region of concentrated $\mathrm{Cu}-\mathrm{Ge}$ and $\mathrm{Cu}-\mathrm{Zn}$ single crystals-I. critical resolved shear stress, slip line formation and the true strain rate," Acta Metall., 25, pp. 437-446.

[14] Hardwick, D. A., 1987, "The mechanical properties of thin films: A review," Thin Solid Films, 154, pp. 109-124.

[15] Standard Methods of Tension Testing of Metallic Foil, Annual Book of ASTM Standards, Vol. 3, ASTM, Philadelphia, pp. 490-495, 1987.

[16] Lawley, A., and Schuster, S., 1962, "Preparation and tension testing of thin metals foils of rolled materials," Rev. Sci. Instrum., 33, pp. 1178-1179.

[17] Hong, H.-K., and Cheng, C. G., 1992, "In-situ microstructural observation and concurrent measurement of load and strain histories," Proceedings of the SEM Seventh International Congress on Experimental Mechanics (ICEM-7), June 8-11, 1992, Las Vegas, Society for Experimental Mechanics, Inc., Bethel, pp. 932-937.

[18] Hong, H.-K., and Liu, C.-S., 2002, "Minkowski spacetime and spinor of double-slip model for planar polycrystal plasticity," submitted for possible publication.

[19] Clement, A., 1982, "Prediction of deformation texture using a physical principle of conservation,” Mater. Sci. Eng., 55, pp. 203-210. 